SUBJECT AREAS:

MOLECULAR

NEUROSCIENCE

NON-CODING RNAS

GENE REGULATION

SCIENTIFIC REPORTS:

$2: 0529$

DOI: $10.1038 /$ srep00529

(2012)

Published:

24 July 2012

Updated:

22 November 2013

\section{ERRATUM: Position-dependent FUS-RNA interactions regulate alternative splicing events and transcriptions}

Shinsuke Ishigaki ${ }^{1,4 *}$, Akio Masuda ${ }^{2 *}$, Yusuke Fujioka', Yohei Iguchi' ${ }^{1}$ Masahisa Katsuno', Akihide Shibata ${ }^{2}$, Fumihiko Urano ${ }^{3}$, Gen Sobue ${ }^{1,4}$ \& Kinji Ohno ${ }^{2}$

\footnotetext{
'Department of Neurology, Nagoya University Graduate School of Medicine, Nagoya, Japan, ${ }^{2}$ Division of Neurogenetics, Center for Neurological Diseases and Cancer, Nagoya University Graduate School of Medicine, Nagoya, Japan, ${ }^{3}$ Program in Gene Function and Expression, University of Massachusetts Medical School, Worcester, MA, ${ }^{4}$ CREST, Japan Science and Technology Agency, Kawaguchi, Japan.
}

The details for affiliation 1 were incorrect in the original HTML version of this Article. The affiliation was incorrectly listed as "Department of Neurology, Center for Neurological Diseases and Cancer, Nagoya University Graduate School of Medicine, Nagoya, Japan". The correct affiliation is listed above. This has now been corrected in the HTML version of the Article. 